Open Access

\title{
Distribution characteristics of salivary cortisol measurements in a healthy young male population
}

Hiromitsu Kobayashi and Yoshifumi Miyazaki

\begin{abstract}
Background: Salivary cortisol has been used in various fields of science as a non-invasive biomarker of stress levels. This study offers the normative reference values of cortisol measurement for healthy young males.

Findings: Salivary cortisol levels were measured in 267 healthy young males (age: $21.7 \pm 1.5$ years) in the early morning on two consecutive days and were analyzed by radioimmunoassay. Frequency distribution analysis was conducted with mean values of the measurements taken on the 2 days. The mean salivary cortisol level was $20.39 \pm 7.74 \mathrm{nmol} / \mathrm{I}$ (median: $19.31 \mathrm{nmol} / \mathrm{l}$ ). The skewness and kurtosis of the distribution of the raw data were 0.72 and 0.68 , respectively. They were both improved by a square root transformation but not by a logarithmic transformation.

Conclusions: The skewness of the distribution for salivary cortisol measured in the early morning is considerably smaller than that previously reported from afternoon measurements. A "floor effect" may be an explanation for the difference in the distribution characteristics of salivary cortisol.
\end{abstract}

Keywords: salivary cortisol, distribution, skewness, kurtosis, floor-effect

\section{Background}

Cortisol is a steroid hormone produced by the zona fasciculata of the adrenal cortex to regulate carbohydrate, fat, and protein metabolism. The cortisol level is considered to be an indicator of the activity of the hypothalamic-pituitary-adrenal (HPA) axis, which is responsible for corticotropin-releasing hormone (CRH) and adrenocorticotropin (ACTH) secretions. The HPA system is among the major stress response systems of the human biology. The ACTH-mediated cortisol secretion induces an increase in blood pressure and glucose levels and immune system suppression. These physiological reactions are called "fight or flight" response. Thus, cortisol measurements have been used as a biological indicator of stress.

Both acute (e.g., skydiving [1]) and chronic (e.g., work overload [2]) stress can lead to elevations in blood levels of cortisol. Cortisol additionally increases due to chronic

\footnotetext{
* Correspondence: ymiyazaki@faculty.chiba-u.jp

Center for Environment, Health and Field Sciences, Chiba University, Chiba,
} Japan
[4], or psychiatric disorders [5].

Chronic exposure to elevated cortisol may cause some pathogenic processes [6]. This cumulative effect of stress responses on physiological functions is called allostatic load [7]. Thus, cortisol is an effective allostasis/allostatic oad indicator [6].

Cortisol level analysis was performed with blood serum. The disadvantage of this method is not only costly but it may also lead to falsely elevated cortisol levels because blood collection alone may activate the HPA axis [8]. On the other hand, cortisol is also detected in hair, urine and saliva. Hair cortisol concentration provides an advantage for evaluating chronic stress because hair growth is slow. Conversely, salivary cortisol levels indicate acute response to stress and serum cortisol [9]. Salivary cortisol concentration was directly proportional to the serum unbound cortisol concentration [10]. The recent collection and analytical protocol development for human salivary cortisol expanded the application range of the measurements. For instance, salivary cortisol is used as a neuroendocrine 
marker of stress in various fields of science, including physiological anthropology $[11,12]$.

Several studies documented salivary cortisol in large populations of healthy individuals [13], including 6470 healthy adults aged 45 years [14] and 1768 children [15]. Thus, most large-scale studies investigated middle-aged adults ( $>40$ years) or pre-adolescent children (10-13 years). However, none of them determined normal cortisol levels in the saliva of young adults (20-30 years). Therefore, the present study determined the normative salivary cortisol values in 267 healthy Japanese males, with special reference to the characteristics of the concentration distribution.

\section{Methods \\ Participants}

The 267 Japanese male students who participated in the present study constitute a subset of the participants enrolled in our previous study on 417 young Japanese male students [16]. None reported physical or psychiatric disorder history. All participants were non-smokers, and alcohol or dietary supplement intake on the day before the measurement was forbidden. The study was conducted under the regulations of the Institutional Ethical Committee of the Forestry and Forest Products Research Institute and the Center for Environment, Health and Field Sciences, Chiba University, Japan.

\section{Saliva collection and analysis}

Saliva samples were collected from each participant using a Salivette (No. 51.1534; Sarstedt, Numbrecht, Germany). The saliva was collected before breakfast, approximately $20-40 \mathrm{~min}$ after awakening (6:30-7:30 a.m.) and before they brushed their teeth. Each participant rested for $1 \mathrm{~min}$ in a sitting position before saliva collection. The measurement was repeated the following day. The samples were immediately frozen and transported to the laboratories of SRL Inc. (Tokyo, Japan) for cortisol concentration measurement. An aliquot of $0.25 \mathrm{ml}$ of saliva was analyzed by radioimmunoassay.

\section{Statistical analysis}

The initial cortisol level (COR-raw) measurements were converted using natural logarithmic (COR-log) and square root (COR-sqrt) scales. The mean value of the measurements taken on two consecutive days from each participant was used to characterize the distribution. The mean, median, standard deviation (SD), coefficient of variation ( $\mathrm{CV} ; \mathrm{SD} /$ mean), skewness (symmetry parameter), kurtosis (peakedness parameter), and $95 \%$ confidence interval $(95 \% \mathrm{CI})$ were calculated for the raw data and numerically transformed cortisol levels.
Table 1 Demographic parameters of the participants

\begin{tabular}{llll}
\hline & Age (years) & Height $(\mathrm{cm})$ & Weight $(\mathrm{kg})$ \\
\hline Mean & 21.8 & 171.9 & 64.4 \\
SD & 1.5 & 5.4 & 9.6 \\
Max & 29 & 188 & 110 \\
Min & 20 & 155 & 47 \\
\hline
\end{tabular}

$S D$ standard deviation

\section{Results}

The participants were healthy young adults (mean, $21.8 \pm$ 1.5 years; range, 20-29) with normal and consistent height (mean, $171.9 \pm 5.4 \mathrm{~cm} ; 155-188 \mathrm{~cm}$ ) and body weight (mean, $64.4 \pm 9.6 \mathrm{~kg}$; range, $47-110 \mathrm{~kg}$ ) (Table 1).

The quantitative analysis of the raw (COR-raw) and numerically transformed (COR-log and COR-sqrt) salivary cortisol levels are summarized in Table 2. Although the COR-raw exhibited $38 \%$ of $\mathrm{CV}$, the numerical transformations reduced the CVs to $14 \%$ (COR-log) and $20 \%$ (COR-sqrt).

The frequency distribution histograms were compared to test the data transformation impact on the interindividual variability in cortisol levels (Fig. 1). The CORraw histogram exhibited a slightly right-skewed and peaked curve, consistent with the skewness of 0.73 and the kurtosis of 0.68 . In contrast, the COR-log histogram was left-skewed because of a skewness of -0.63 and presented a kurtosis of 1.14. Interestingly, the square root conversion generated a nearly normal distribution because the COR-sqrt histogram only exhibited skewness and kurtosis values of 0.12 and 0.20 , respectively.

\section{Discussion}

Cortisol secretion follows a circadian rhythm, whereby circulating levels are low or undetectable at midnight, accumulate overnight to peak 30-45 min after awakening, and then decline slowly throughout the day [8]. This peak phenomenon is called cortisol awakening

Table 2 Characteristics of the distribution of the raw and numerically transformed salivary cortisol levels in healthy young male adults

\begin{tabular}{llll}
\hline & COR-raw $(\mathrm{nmol} / \mathrm{l})$ & COR-log $\ln (\mathrm{nmol} / \mathrm{l})$ & COR-sqrt $(\mathrm{nmol} / \mathrm{l})$ \\
\hline Mean & 20.39 & 2.92 & 4.41 \\
Median & 19.31 & 2.94 & 4.37 \\
$\mathrm{SD}$ & 7.74 & 0.41 & 0.86 \\
$\mathrm{CV}$ & $38 \%$ & $14 \%$ & $20 \%$ \\
$95 \% \mathrm{Cl}$ & $7.59-39.59$ & $1.99-3.67$ & $2.72-6.28$ \\
Skewness & 0.73 & -0.63 & 0.12 \\
Kurtosis & 0.68 & 1.14 & 0.20
\end{tabular}

The skewness and kurtosis of a normal distribution are both zero $S D$ standard deviation, $C V$ coefficient of variation (SD/mean), $C l$ confidence interval, skewness parameter of symmetry, kurtosis parameter of peaked (positive) or flat (negative) distribution 

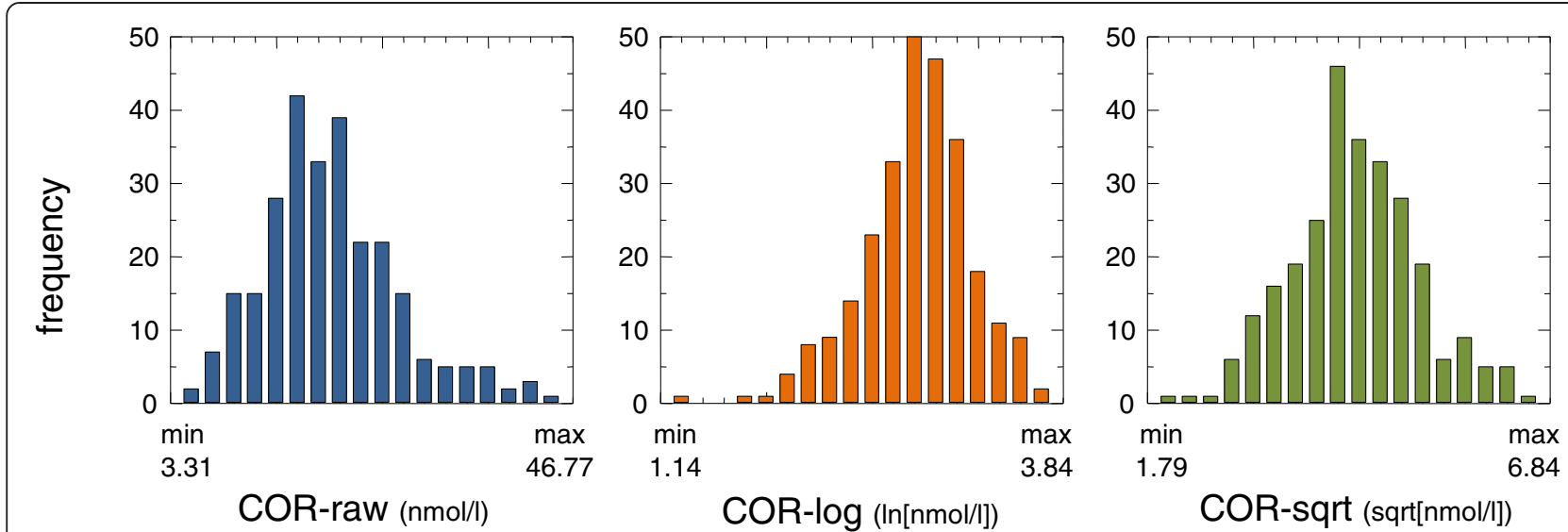

Fig. 1 Frequency distributions of the raw and numerically transformed salivary cortisol levels of healthy young male adults. The raw data (COR-raw) exhibit a right-skewed distribution, whereas the logarithmic data (COR-log) exhibit a left-skewed distribution. In contrast, the square root transformation (COR-sqrt) generated a nearly symmetrical distribution

response (CAR). Thus, samples collected in the early morning provide the most sensitive measurements of cortisol levels. The present study shows that saliva samples collected from healthy young Japanese adults in the early morning contain a mean cortisol level of $20.39 \mathrm{nmol} / \mathrm{l}$. These results are mostly consistent with the mean salivary cortisol level measured in the early morning in 45-year-old men in England (21.01 nmol/l) [14] and in elderly subjects in Rotterdam ( $<65$ years; $18.4 \mathrm{nmol} / \mathrm{l}$ ) [17]. Furthermore, Hansen et al. [18] reported a $95 \%$ CI for salivary cortisol level of 7.6$39.4 \mathrm{nmol} / \mathrm{l}$ in the early morning for adults in Denmark, which is remarkably similar to the $95 \%$ CI calculated in the present study (7.59-39.59). Collectively, these results demonstrate that salivary cortisol level measurements in the early morning are highly consistent, even between adult populations of distinct ethnicities.

Mean height and body mass of the participants were almost identical with the means of Japanese males aged 22 years $(171.3 \pm 4.7 \mathrm{~cm}$ and $65.0 \pm 10.7$ $\mathrm{kg}$ ) [19]. Therefore, the sample population of this study can be considered as representatives of physical traits. For socioeconomic status, the sample population may not be a good representative of the Japanese population because all participants were college students. This is a limitation in interpreting the present results.

Little is known about the distribution characteristics of salivary cortisol levels in large populations. The present study demonstrates that the skewness of the data distribution for salivary cortisol measured in the early morning is considerably smaller (skewness, 0.73 ) than for afternoon measurements (skewness, 3.5-4.8) that previously reported [20, 21]. Kiess et al. [22] investigated the diurnal change of salivary cortisol in various age groups. The median of the morning cortisol was located in approximately the middle of the $90 \%$ interval, suggesting an approximate symmetrical distribution. Conversely, evening cortisol median shifted close to the fifth percentile value, suggesting a right-skewed distribution. The median shift toward the lower limit of the variation was consistently observed in adults, adolescents, children, and neonates. Similar results were additionally demonstrated in children (mean age $=10.9$ years) by Carrion et al. [23] . Thus, the difference in skewness could be attributed to measurement time of day, rather than differences in age, gender, or ethnicity.

Even if the distribution of peak cortisol in the early morning is almost symmetrical, the lowered cortisol level in the afternoon does not necessarily present a symmetrical distribution. A "floor effect" [24] prohibits negative values in cortisol, resulting in a distorted distribution with a shorter left tail (right skewness). This phenomenon may explain the difference in skewness of the data distributions obtained with afternoon and morning samples.

In conclusion, the present study demonstrated that the skewness of salivary cortisol concentration measured in the early morning is relatively smaller compared with that measured in the afternoon. Although logarithmic transformations have been proposed for salivary cortisol in the previous studies [13, 18, 21], we recommend a square root transformation for morning salivary cortisol measurements.

\section{Abbreviations}

ACTH: adrenocorticotropin; CAR: cortisol awakening response; $\mathrm{Cl}$ : confidence interval; COR-log: natural logarithmic transformed salivary cortisol; COR-raw: raw salivary cortisol; COR-sqrt: square root transformed 
salivary cortisol; $\mathrm{CRH}$ : corticotropin-releasing hormone; $\mathrm{CV}$ : coefficient of variation; HPA: hypothalamic-pituitary-adrenal; SD: standard deviation.

\section{Competing interests}

The authors declare that they have no competing interests.

\section{Authors' contributions}

HK performed statistical analysis and data interpretation. YM had the overall responsibility of the study design. Both authors read and approved the final manuscript.

\section{Acknowledgements}

This study was supported by the 2015 Strategic Innovation Promotion (SIP) Program of the National Agriculture and Food Research Organization (NARO) in Japan.

Received: 10 April 2015 Accepted: 29 July 2015

Published online: 19 August 2015

\section{References}

1. Chatterton Jr RT, Vogelsong KM, Lu YC, Hudgens GA. Hormonal responses to psychological stress in men preparing for skydiving. J Clin Endocrinol Metab. 1997:82:2503-9.

2. Schulz P, Kirschbaum C, Prubner J, Hellhammer D. Increased free cortisol secretion after awakening in chronically stressed individuals due to work overload. Stress Med. 1998;14:91-7.

3. Chiodini I, Adda G, Scillitani A, Coletti F, Morelli V, Di Lembo S, et al. Cortisol secretion in patients with type 2 diabetes relationship with chronic complications. Diabetes Care. 2007;30:83-8.

4. Raff H, Raff JL, Findling JW. Late-night salivary cortisol as a screening test for Cushing's syndrome. J Clin Endocrinol Metab. 1998;83:2681-6.

5. McBurnett K, Lahey BB, Frick PJ, Risch C, Loeber R, Hart EL, et al. Anxiety, inhibition, and conduct disorder in children: II. Relation to salivary cortisol. J Am Acad Child Adolesc Psychiatry. 1991;30:192-6.

6. Dowd JB, Simanek AM, Aiello AE. Socio-economic status, cortisol and allostatic load: a review of the literature. Int J Epidemiol. 2009;38:1297-309.

7. Seeman TE, Singer BH, Rowe JW, Horwitz RI, McEwen BS. Price of adaptation - allostatic load and its health consequences: MacArthur studies of successful aging. Arch Intern Med. 1997;157:2259-68.

8. Kirschbaum C, Hellhammer DH. Salivary cortisol. Encyclopedia Stress. 2000:3:379-83.

9. Lee do Y, Kim E, Choi MH. Technical and clinical aspects of cortisol as a biochemical marker of chronic stress. BMB Rep. 2015;48:209-16.

10. Vining RF, McGinley RA, Maksvytis JJ, Ho KY. Salivary cortisol: a better measure of adrenal cortical function than serum cortisol. Ann Clin Biochem. 1983;20:329-35.

11. Squires EC, McClure HH, Martinez CR, Eddy JM, Jiménez RA, Isiordia LE, et al. Diurnal cortisol rhythms among Latino immigrants in Oregon. USA J Physiol Anthropol. 2012;31:19.

12. Logan AC, Felice N, Jacka FN. Nutritional psychiatry research: an emerging discipline and its intersection with global urbanization, environmental challenges and the evolutionary mismatch. J Physiol Anthropol. 2014;33:22.

13. Adam EK, Kumari M. Assessing salivary cortisol in large-scale, epidemiological research. Psychoneuroendocrinology. 2009;34:1423-36.

14. Power C, Li L, Hertzman C. Associations of early growth and adult adiposity with patterns of salivary cortisol in adulthood. J Clin Endocrinol Metab. 2006:91:4264-70.

15. Rosmalen JG, Oldehinkel AJ, Ormel J, de Winter AF, Buitelaar JK, Verhulst FC. Determinants of salivary cortisol levels in 10-12 year old children; a population-based study of individual differences. Psychoneuroendocrinology. 2005;30:483-95.

16. Kobayashi H, Park BJ, Miyazaki Y. Normative references of heart rate variability and salivary alpha-amylase in a healthy young male population. J Physiol Anthropol. 2012;31:9.

17. Hek K, Direk N, Newson RS, Hofman A, Hoogendijk WJ, Mulder CL, et al. Anxiety disorders and salivary cortisol levels in older adults: a populationbased study. Psychoneuroendocrinology. 2013;38:300-5.

18. Hansen $\AA$ M, Garde AH, Christensen JM, Eller NH, Netterstrøm B. Evaluation of a radioimmunoassay and establishment of a reference interval for salivary cortisol in healthy subjects in Denmark. Scand J Clin Lab Invest. 2003;63:303-10.
19. Ministry of Health, Labour and Welfare of Japan. National Health and Nutrition Survey 2013. http://www.mhlw.go.jp/bunya/kenkou/eiyou/dl/h25houkoku.pdf. Accessed 21 Jun 2015.

20. Smider NA, Essex MJ, Kalin NH, Buss KA, Klein MH, Davidson RJ, et al. Salivary cortisol as a predictor of socioemotional adjustment during kindergarten: a prospective study. Child Dev. 2002;73:75-92.

21. Gordis EB, Granger DA, Susman EJ, Trickett PK. Salivary alpha amylasecortisol asymmetry in maltreated youth. Horm Behav. 2008;53:96-103.

22. Kiess W, Meider A, Dressendorfer RA, Schriever K, Kessler U, Konig A, et al. Salivary cortisol levels throughout childhood and adolescence: relation with age, pubertal stage, and weight. Pediatr Res. 1995;37:502-6.

23. Carrion VG, Weems CF, Ray RD, Glaser B, Hessl D, Reiss AL. Diurnal salivary cortisol in pediatric posttraumatic stress disorder. Biol Psychiatry. 2002;51:575-82.

24. Cohen N, Flamenbaum W. Obesity and hypertension. Demonstration of a "floor effect". Am J Med. 1986;80:177-81.81.

\section{Submit your next manuscript to BioMed Central and take full advantage of:}

- Convenient online submission

- Thorough peer review

- No space constraints or color figure charges

- Immediate publication on acceptance

- Inclusion in PubMed, CAS, Scopus and Google Scholar

- Research which is freely available for redistribution 